\title{
Atuação da enfermagem em trabalho remoto no contexto da pandemia COVID-19
}

RESUMO | Objetivo: relatar a experiência de desenvolvimento do trabalho remoto pela equipe de enfermagem, sua sistematização e desafios, durante a pandemia da COVID-19. Método: trata-se de um estudo de natureza descritiva, exploratória, com abordagem qualitativa, do tipo relato de experiência, realizado em um Hospital Universitário Federal de Minas Gerais. Relato baseado na experiência de enfermeiras frente à realização de trabalho remoto no contexto da pandemia COVID-19, no período de março a julho de 2020. Resultados: colaboradores em trabalho remoto formaram a segunda linha de enfretamento à COVID-19, executando protocolos assistenciais, instruções técnicas de trabalho, materiais educativos, desenvolvimento de tecnologias leves em saúde para educação de profissionais e pacientes e telemonitoramento de pacientes. Conclusão: o trabalho remoto é uma inovação no processo de trabalho da enfermagem ampliando as perspectivas do cuidado e garantindo a segurança dos profissionais dos grupos de risco.

Palavras-chaves: Coronavírus; Pandemia; Cuidados de Enfermagem.

ABSTRACT | Objective: to report the experience of developing remote work by the nursing team, its systematization and challenges, during the COVID-19 pandemic. Method: this is a descriptive, exploratory study with a qualitative approach, an experience report type, carried out in a Federal University Hospital in Minas Gerais. Report based on the experience of nurses regarding the performance of remote work in the context of the pandemic COVID-19, from March to July 2020. Results: collaborators in remote work formed the second line of confrontation to COVID-19, executing care protocols, technical work instructions, educational materials, development of light health technologies for the education of professionals and patients and telemonitoring of patients. Conclusion: remote work is an innovation in the nursing work process, expanding the perspectives of care and ensuring the safety of professionals from risk groups.

Keywords: Coronavirus; Pandemics; Nursing Care.

RESUMEN | Objetivo: informar la experiencia de desarrollar trabajo remoto por parte del equipo de enfermería, su sistematización y desafíos, durante la pandemia de COVID-19. Método: se trata de un estudio exploratorio descriptivo con enfoque cualitativo, un tipo de informe de experiencia, realizado en un Hospital Universitario Federal en Minas Gerais. Informe basado en la experiencia de las enfermeras sobre el desempeño del trabajo remoto en el contexto de la pandemia COVID-19, de marzo a julio de 2020. Resultados: los colaboradores en el trabajo remoto formaron la segunda línea de confrontación con COVID-19, ejecutando protocolos de atención, instrucciones de trabajo técnico, materiales educativos, desarrollo de tecnologías de salud ligera para la educación de profesionales y pacientes y telemonitorización de pacientes. Conclusión: el trabajo remoto es una innovación en el proceso de trabajo de enfermería, que amplía las perspectivas de atención y garantiza la seguridad de los profesionales de los grupos de riesgo. Palavras claves: Coronavirus; Pandemias; Atención de Enfermería.

\section{Maria Fernanda Silveira Scarcella}

Enfermeira Cardiologista da Clínica Médica Hospitalar do Hospital das Clínicas da Universidade Federal de Minas Gerais (HCUFMG/EBSERH). Mestre em Enfermagem pela Escola de Enfermagem/UFMG.

ORCID: 0000-0002-3319-1646

\section{Pamela Nery do Lago}

Enfermeira e Coordenadora de Enfermagem da Clínica Médica Hospitalar do HC-UFMG/ EBSERH. Especialista em Enfermagem ClínicoCirúrgica, em Enfermagem Oncológica e em Gestão Hospitalar. Mestranda em Gestão de Cuidados da Saúde.

ORCID: 0000-0002-3421-1346

Recebido em: 22/07/2020

Aprovado em: 27/07/2020

INTRODUÇÃO

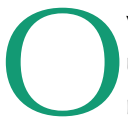
vírus SARS-CoV-2, trata-se um novo subtipo de coronavírus que surgiu no final de 2019, responsável pela doença COVID-19 ${ }^{(1)}$. Em 30 de janeiro de 2020 a Organização Mundial de Saúde (OMS) decretou emergência de saúde pública de importância internacional(2) e uma pandemia no dia 11 de março de $2020^{(3)}$. Segundo dados de abril de 2020, 210 países contaram com 2,1 milhão de casos confirmados e mais de 144 mil mortes ${ }^{(4)}$.

Dentre os grupos de maior vulnerabilidade para a doença, estão os trabalhadores dos serviços de saúde com alto risco para contaminação, e representaram uma parcela expressiva do número de ca$\operatorname{sos}^{(5)}$. Os indicadores sobre a exposição e o contágio, de letalidade e de morbidade da COVID-19 entre os trabalhadores da saúde, indicam em vários países do mundo a variação entre 4 e $12 \%$ dos casos notificados $^{(6)}$.

O Brasil, até o início de maio de 2020, tinha 31.790 casos confirmados em profissionais de saúde. No total, 199.768 foram identificados como casos suspeitos e precisaram ser afastados, sendo os mais afetados: técnicos ou auxiliares de enfermagem $(34,2 \%)$, enfermeiros $(16,9 \%)$ e médicos $(13,3 \%)^{(7)}$.

O Conselho Nacional de Saúde no Parecer Técnico $n^{\circ}$ 128/2020, orienta o trabalho dos profissionais de saúde durante a pandemia da COVID-19, recomendando que os gestores da saúde devem assegurar como direito aos profissionais da saúde o exercício do trabalho em locais seguros quando estes estiverem em condições de risco à vida ou saúde, 
como no caso de profissionais em situações de maior vulnerabilidade ${ }^{(6)}$.

Diante da pandemia, o alto risco de contaminação dos profissionais de saúde e a suscetibilidade de muitos destes devido às vulnerabilidades preexistentes, tornou-se necessário o afastamento dos postos de trabalho, e nova modalidade de exercício da enfermagem foi necessária ser empregada, em que os profissionais mantenham-se em isolamento social e, dando continuidade as atividades inerentes à prática da enfermagem necessárias ao enfretamento da pandemia, bem como, garantindo a segurança dos mesmos.

Nesse interim temos o chamado trabalho remoto em enfermagem, que trata-se de uma inovação no processo laboral e uma faceta na transformação das relações de trabalho e assistência aos pacientes que emergiu mediante a pandemia da COVID-19.

Nesse contexto, este estudo tem por objetivo relatar a experiência do desenvolvimento de trabalho remoto pela equipe de enfermagem de um hospital público federal, sua sistematização e desafios, durante a pandemia da COVID-19.

\section{MÉTODO}

Trata-se de um estudo descritivo, exploratório, com abordagem qualitativa, do tipo relato de experiência, realizado em um Hospital Universitário Federal da cidade de Belo Horizonte, Minas Gerais. $\mathrm{O}$ relato foi baseado na experiência de enfermeiras frente à realização de trabaIho remoto no contexto da pandemia da COVID-19, no período de março a julho de 2020.

Realizou-se embasamento teórico da literatura por se tratar de temática emergente. Optou-se pelo levantamento do estado da arte em bases de dados nacionais e internacionais para melhor compreensão e fundamentação acerca do tema.

O levantamento de artigos foi realizado em julho de 2020 na Biblioteca Virtual em Saúde (BVS) direcionando as buscas nas bases de dados: Medical Literature Analysis and Retrieval System Online (MEDLINE), Literatura Latino-americana e do Caribe em Ciências da Saúde (LILACS), Base de Dados em Enfermagem (BDENF) e Pubmed, tendo como critérios de inclusão: artigos completos, publicados de janeiro a julho de 2020, disponíveis nos idiomas português, inglês ou espanhol. Para tal foram utilizados os descritores indexados: coronavírus; pandemia; cuidados de enfermagem.

Devido à temática ser nova e com construção de evidências recentes não foi excluída nenhuma publicação. As informações extraídas dos artigos foram compiladas em um instrumento de coleta de dados construído pelas próprias pesquisadoras contento: título do artigo, base de dados indexada, autor, país de origem, idioma, ano de publicação, local de realização da pesquisa, métodos, resposta a questão norteadora e principais conclusões.

A experiência foi desenhada a partir da observação direta das pesquisadoras em seu ambiente de trabalho. Por se tratar de um relato de experiência, não houve aplicação de termo de consentimento livre e esclarecido.

\section{RESULTADOS: relato de experiência}

\section{tornou-se necessário o afastamento dos postos de trabalho}

As instituições de saúde brasileiras se depararam em março de 2020 com um desafio, a pandemia da COVID-19, colocando os serviços de saúde do mundo em risco de colapso devido à alta demanda por assistência dos indivíduos acometidos pela doença. Em 16 de abril de 2020, já tínhamos no Brasil uma incidência de 14,51 casos por 100 mil habitantes ${ }^{(8)}$.

A doença em pouco tempo avançou por todos os continentes. Os profissionais de saúde, por comporem a linha de frente de enfrentamento à pandemia, foram acometidos de forma significativa em vários países pelo mundo. Dentre as classes de profissionais de saúde do Brasil, a com maior número de casos e óbitos, foram de trabalhadores da equipe de enfermagem. 
Visando a proteção ocupacional, linhas de enfrentamento foram dispostas na literatura internacional, e no Brasil, por meio de deliberações governamentais previu-se que profissionais de saúde com maior vulnerabilidade para casos fatais da doença fossem afastados dos locais de trabalho e das atividades que lidassem de forma direta com o novo coronavírus.

Dessa forma, instituição federal, pública, de assistência à saúde, seguindo tais recomendações, afastou dentre os seus colaboradores àqueles do chamado grupo de risco, ou seja, trabalhadores imunossuprimidos, com doenças crônicas, gestantes etc., que têm maior predisposição a desfechos fatais se acometidos pela COVID-19. Nesse contexto cerca de 280 profissionais de enfermagem foram afastados para trabalho remoto.

Surge nesse momento um novo desafio para a enfermagem: como assistir aos pacientes, mediante a uma crise sanitária mundial, onde recursos humanos em saúde estariam escassos, e ao mesmo tempo manter o necessário isolamento social para segurança dos trabalhadores? E ainda: como se alicerçaria o trabalho remoto em enfermagem, mantendo, mesmo que remotamente, sua essência que é o cuidado aos pacientes, e mais, nesse momento onde o cuidado em saúde em uma situação de emergência e de alta qualidade se fazia necessário?

A pandemia expôs fragilidades e lacunas do serviço de saúde de todo o mundo e precipitou a necessidade urgente de adaptação destes para o enfretamento e manutenção de uma assistência de qualidade.

Os colaboradores em trabalho remoto foram estrategicamente colocados como segunda linha de enfretamento à COVID-19, contribuindo com a execução de protocolos assistenciais, instruções técnicas de trabalho (ITTs), materiais educativos, desenvolvimento de tecnologias leves para educação de profissionais e pacientes, telemonitoramento de pacientes.

A organização do trabalho remoto se deu a partir da divisão dos trabalhadores em times, os quais eram sempre liderados por enfermeiras. A alocação dos profissionais por time teve como critério a área de experiência e atuação, assim houveram times de terapia intensiva, clínica médica, hemodiálise, maternidade etc.

Em um primeiro momento, os profissionais em trabalho remoto ficaram

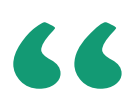

A pandemia expôs eram atividades prioritárias. fragilidades e lacunas do serviço de saúde de todo o mundo e precipitou a necessidade urgente de adaptação destes para o enfretamento e manutenção de uma assistência de qualidade.

responsáveis pela elaboração dos protocolos de manejo da COVID-19, material educativo, treinamentos à distância e instruções de trabalho que envolvia a doença. Esse processo foi de fundamental importância para o serviço de saúde, visto que por se tratar de uma doença emergente, os profissionais de saúde pos-
Poder contar com os trabalhadores

suíam pouco ou nenhum conhecimento teórico e técnico sobre a doença, em um momento em que a necessidade destes era urgente.

Em seguida, os times de trabalho remoto estenderam suas atividades para elaboração e atualização de outros protocolos e ITTs da instituição. Todos os documentos que subsidiam e sistematizam as ações assistenciais do hospital foram atualizados, demonstrando uma alta produtividade desses times e grande contribuição, visto que apesar de essenciais para o cuidado, no atual momento, não em trabalho remoto permitiu que atividades administrativas que envolvem o cuidado de enfermagem e que viabilizam a assistência direta, em um momento em que todos os esforços da instituição estavam voltados para a reorganização do cuidado clínico aos pacientes infectados, foi crucial e de extremo benefício para a qualidade e segurança da assistência prestada aos pacientes.

\section{DISCUSSÃO}

Os profissionais de saúde têm alto risco de contaminação na cadeia epidemiológica da doença, devido à exposição ocupacional constante para dar resposta às demandas da pandemia, além de muitas vezes estarem sob condições de trabalho insatisfatórias para prevenção de transmissão cruzada ${ }^{(9 ; 10)}$.

Com objetivo de diminuir a contaminação dos profissionais de saúde, faz-se mister que profissionais vulneráveis sejam afastados da assistência direta à COVID-19, tais como: gestantes; idosos; indivíduos com histórico de doenças crônicas ou doenças auto-imunes ${ }^{(11)}$.

Corroborando com o exposto, compreende-se que as instituições de saúde devem garantir a adoção de medidas e mecanismos de proteção e promoção à saúde para todos os trabalhadores que atuam nos seus serviços ${ }^{(5)}$.

O Brasil é o país onde mais morrem 
profissionais de enfermagem por COVID-19: 143 profissionais vieram a óbito e há 16.064 casos confirmados, segundo dados de junho de 2020 do Conselho Federal de Enfermagem ${ }^{(12)}$.

Dentre as principais medidas, emergiu o trabalho remoto como uma inovação das atividades laborais. Nesse contexto os colaboradores exercem suas funções à distância, lançando mão de teleconferências, vídeo-aulas e outras ferramentas de comunicação e execução de produtividade para assistência à saúde. Trata-se de uma inovação tecnológica para resolver os problemas temporários que surgiram devido à pandemia ${ }^{(13)}$.

Devido a COVID-19 ser uma doença emergente, todo o fluxo assistencial institucional necessitou ser sistematizado e reorganizado em caráter emergencial para prover aos trabalhadores da linha de frente bases seguras, por meio de protocolos e instruções técnicas, para o manejo da COVID-19, assim estes poderiam concen- trar seus esforços na assistência direta aos pacientes, enquanto a equipe de enfermagem em trabalho remoto forneceria as bases educacionais e administrativas para permitir um cuidado seguro em saúde.

Tal feito garantiu subsídios para que os profissionais da linha de frente tivessem embasamento técnico e científico constante para o manejo clínico da COVID-19, além de oportunizar a manutenção da organização de toda a educação permanente hospitalar, essencial ao processo seguro de cuidar, mas que poderia ser deixada em segundo plano devido à súbita necessidade de transformação e reorganização da assistência para atender a demanda da COVID-19.

\section{CONCLUSÃO}

O trabalho remoto trata-se de uma inovação na prática da enfermagem e na área da saúde e, portanto um desafio aos profissionais.
Entendemos que sua execução oportunizou uma melhor organização do serviço de saúde em uma situação de crise, e sua atuação como segunda linha de enfrentamento da pandemia COVID-19, permitiu que as partes envolvidas no cuidado aos pacientes pudessem assisti-los de forma segura e com qualidade.

A experiência do trabalho remoto na enfermagem foi benéfica para a instituição, já que fortaleceu a educação permanente e permitiu que outras atividades administrativas que ficaram prejudicadas devido à pandemia, pudessem continuar acontecendo; para os profissionais de saúde da linha de frente, que puderam ser treinados e dispuseram de provimento contínuo teórico e prático para embasamento do seu exercício em evidências científicas, garantindo segurança para si e para os pacientes; e para os profissionais em trabalho remoto, que produziram e atenderam as necessidades dos citados.

\section{Referências}

1. Rubin EJ, et al. Audio Interview: Practical Measures to Help Prevent Covid-19. N Engl J Med [Internet] 2020 [acessado em 17 de jul de 2020]; 382(13): e32. Disponivel em https://www.nejm.org/doi/full/10.1056/nejme2006742

2. WORLD HEALTH ORGANIZATION (WHO). WHO Director-General's statement on IHR Emergency Committee on Novel Coronavirus (2019-nCoV) Geneva:WHO; 2020. [acessado em 28 de jul de 2020]. Disponível em https:/l www.who.int/news-room/detail/23-01-2020-statement-on-the-meeting-of-the-international-health-regulations-(2005)-emergency-committee-regarding-the-outbreak-of-novel-coronavirus-(2019-ncov).

3. WORLD HEALTH ORGANIZATION (WHO). WHO Director-General's opening remarks at the media briefing on COVID-19-11 March 2020 Geneva: WHO; 2020 [acessado em 28 de jul de 2020]. Disponível em https://www. who.int/dg/speeches/detail/who-director-general-s-opening-remarks-at-the-media-briefing-on-covid-19---11-march-2020.

4. WORLDOMETER. Countries where COVID-19 has spread 2020 [acessado em 28 de jul de 2020]. Disponivel em https://www.worldometers.info/coronavirus/countries-where-coronavirus-has-spread/.

5. BRASIL. MINISTÉRIO DA SAÚDE. Secretaria de Vigilância em Saúde. Recomendações de proteção aos trabalhadores dos serviços de saúde no atendimento de COVID-19 e outras síndromes gripais. [publicação online]; 2020 [acesso em 20 abr 2020]. Disponivel em https://www.saude.go.gov.br/files/ banner_coronavirus/GuiaMS-Recomendacoesdeprotecaotrabalhadores-COVID-19.pdf

6. CONSELHO NACIONAL DE SAÚDE. Recomendação $n^{\circ} 020$. [publicação online]; 2020 [acesso em 15 jun 2020]. Disponível em http://conselho.saude. gov.br/recomendacoes-cns/1103-recomendac-a-o-no-020-de-07-de-abril-de-2020.

7. BRASIL. MINISTÉRIO DA SAÚDE. Secretaria de Vigilância em Saúde. Boletim epidemiológico: Doença pelo Coronavírus COVID-19. [publicação on- line]; 2020 [acesso em 17 jul 2020]. Disponível em http://saude.gov.br/images/pdf/2020/July/08/Boletim-epidemiologico-COVID-21-corrigido-13h35. pdf

8. REDE COVIDA [Internet]. Painel Coronavírus Brasil Salvador: Rede CoVida 2020 [acessado em 28 de jul de 2020]. Disponível em http://www.covid19br. org.

9. Zhou M. et al. Knowledge, attitude and practice regarding COVID-19 among health care workers in Henan, China. J Hosp Infect [revista em Internet] 2020 [acessado em 15 de jul de 2020]; 105(2). Disponível em https://www.journalofhospitalinfection.com/article/S0195-6701(20)30187-0/ fulltext.

10. Huh S. How to train the health personnel for protecting themselves from novel coronavirus (COVID-19) infection during their patient or suspected case care. J Educ Eval Health Prof [revista em Internet] 2020 [acessado em 15 de jul de 2020]; 17(10). Disponivel em https://pubmed.ncbi.nlm.nih. gov/32150796/.

11. Li T. Diagnosis and clinical management of severe acute respiratory syndrome Coronavirus 2 (SARS-CoV-2) infection: an operational recommendation of Peking Union Medical College Hospital (V2.0). Emerg Microbes Infect [revista em Internet] 2020 [acessado em 15 de jul de 2020]; 9(1). Disponível em https://www.ncbi.nlm.nih.gov/pmc/articles/PMC7103730/.

12. PORTAL PUBMED [Internet]. Covid-19: Brasil é o país onde mais morrem enfermeiros no mundo por conta da pandemia. 2020 [acessado em 28 de jul de 2020]. Disponível em https://pebmed.com.br/covid-19-brasil-e-o-pais-onde-mais-morrem-enfermeiros-no-mundo-por-conta-da-pandemia/ 13. Aveni A. Estratégias pelo trabalho no futuro devidos a pandemia COVID-19. Revista Processus de Políticas Públicas e Desenvolvimento Social [revista em Internet] 2020 [acessado em 16 de jul de 2020]; [S.I.] 2(3). Disponivel em http://periodicos.processus.com.br/index.php/ppds/article/ view/187. 